\title{
Enabling precision coordinate metrology for universal optical testing and alignment applications (Erratum)
}

\author{
Manal Khreishi, ${ }^{\text {a,b,* }}$ Raymond G. Ohl, ${ }^{\text {a }}$ Joseph M. Howard, ${ }^{\text {a }}$ \\ Jonathan C. Papa, ${ }^{a}$ Ryan McClelland, ${ }^{\text {a Clark Hovis, }}$, \\ Theodore Hadjimichael, ${ }^{a}$ Patrick Thompson, ${ }^{a}$ Kenneth Ranson, ${ }^{a}$ \\ Rongguang Liang, ${ }^{\mathrm{b}}$ and Nicolas Gorius ${ }^{\mathrm{a}, \mathrm{c}}$ \\ ${ }^{a}$ NASA Goddard Space Flight Center, Greenbelt, Maryland, United States \\ ${ }^{b}$ The University of Arizona, College of Optical Sciences, Tucson, Arizona, United States \\ ${ }^{\mathrm{c} C}$ Catholic University of America, Washington, DC, United States
}

[DOI: 10.1117/1.OE.60.4.049801]

This article [Opt. Eng. 60(3), 035106 (15 March 2021) https://doi.org/10.1117/1.OE.60.3 .035106] was originally published with an error in Fig. 56. The RMS error units in parts (a) and (b) were in "nm" instead of " $\mu$ m." The paper was corrected on 7 April 2021.

*Address all correspondence to Manal Khreishi, manal.a.khreishi@nasa.gov 